\title{
Difficulty in Breathing with Decreased Urine Output in São Paulo, Brazil
}

\author{
Marcelino de Souza Durão, Jr. ${ }^{a}$ \\ Debator: Marcelino de Souza Durão, Jr. ${ }^{a}$ \\ Coordinators: Bertrand L. Jaber ${ }^{b}$ Oscar Fernando Pavão dos Santos ${ }^{a}$ \\ antensive Care Unit, Hospital Israelita Albert Einstein, and Nephrology Division, Universidade Federal de São Paulo, \\ São Paulo, Brazil; 'bCaritas St. Elizabeth's Medical Center and Tufts University School of Medicine, Boston, Mass., USA
}

\section{Case Presentation}

\section{Marcelino de Souza Durão, Jr.}

Intensive Care Unit, Hospital Israelita Albert Einstein, and Nephrology Division, Universidade Federal de São Paulo, São Paulo, Brazil

Dr. de Souza Durão: A 57-year-old male was admitted to the hospital complaining of fatigue and difficulty in breathing for the past day.

A nephrological consultation was requested due to acute kidney injury (AKI) and oliguria.

The patient was feeling well until 8 days previously when he developed a fever, a cough and expectoration. At that time, his doctor established an outpatient treatment with intramuscular ceftriaxone and oral moxifloxacin due to a diagnosis of pneumonia. The patient, who is a married diplomat residing in the city of São Paulo, Brazil, did not report any recent travel or contact with sick people.

The patient has a history of hypertension and had a radical prostatectomy 3 years ago due to adenocarcinoma of the prostate. The urological control prior to admission was normal. The patient was currently taking amlodipine and atenolol.

\section{KARGER \\ Fax +4161306 1234 E-Mail karger@karger.ch} www.karger.com (c) 2010 S. Karger AG, Basel

$1660-2110 / 10 / 1164-0274 \$ 26.00 / 0$

Accessible online at: www.karger.com/nec
At the time of admission, the patient's temperature was $36.8^{\circ} \mathrm{C}$. His pulse was 72 beats per minute and blood pressure was $120 \times 65 \mathrm{~mm} \mathrm{Hg}$. His respiratory rate was 28 breaths per minute and oxygen saturation was $88 \%$ at ambient air. Pulmonary auscultation revealed rales in both pulmonary bases, which were more intense in the right lung. After $2 \mathrm{~h}$ of hospitalization, the patient was transferred to the intensive care unit (ICU) with respiratory failure where he was intubated (with the presence of hematic fluid on the orotracheal tube), placed under mechanical ventilation and implanted with a central venous catheter and an invasive arterial pressure monitoring system. Empirical antibiotic therapy with vancomycin, imipenem and clarithromycin was instituted.

The initial cardiac evaluation showed a normal echocardiogram and an absence of variation of the diameter of the inferior vena cava. The cardiac markers (troponin and $\mathrm{CK}-\mathrm{MB}$ ) were normal. Lactate levels were normal, as was the central venous oxygen saturation. The central venous pressure was $20 \mathrm{~mm} \mathrm{Hg}$, and there were no significant variations in the arterial pressure curve during the respiratory cycle.

The computed tomography (CT) of the thorax (without contrast) showed multiple foci of sparse alveolar con-

Marcelino de Souza Durão, Jr., MD

Hospital Israelita Albert Einstein and Federal University of São Paulo

Av. Albert-Einstein 627

São Paulo, 06715510 (Brazil)

Tel. +55 113747 1500, Fax +55 113746 9411, E-Mail marcelino@ einstein.br 
densations primarily affecting the right lung and associated with infiltrates with a ground-glass appearance. There was a small bilateral pleural effusion.

The initial exams showed a leukocyte count of 12,200 cells/ $\mu \mathrm{l}$ (70.8\% neutrophils without a left shift, 13\% lymphocytes, $14 \%$ monocytes and $2 \%$ eosinophils), a hemoglobin level of $9.0 \mathrm{~g} / \mathrm{dl}(13.1 \mathrm{~g} / \mathrm{dl} 8$ days prior), a hematocrit level of $26.1 \%$ and 103,000 platelets/ $\mu l(217,000 / \mu l 8$ days prior). The arterial blood gas revealed a $\mathrm{pH}$ of 7.31. The $\mathrm{pCO}_{2}$ was $31.9 \mathrm{~mm} \mathrm{Hg}$; $\mathrm{pO}_{2}$ was $77.6 \mathrm{~mm} \mathrm{Hg}\left(\mathrm{PO}_{2} / \mathrm{FiO}_{2}\right.$ ratio $=129) . \mathrm{HCO}_{3}$ was $16 \mathrm{mEq} / \mathrm{l}$, and $\mathrm{BE}$ was $-9.2 \mathrm{mEq} / 1$. Renal function was also altered: the creatinine level was $5.8 \mathrm{mg} / \mathrm{dl}(1.3 \mathrm{mg} / \mathrm{dl} 8$ days prior with a glomerular filtration rate estimated by the MDRD study equation at approximately $60 \mathrm{ml} / \mathrm{min}$ at that moment) and the urea level was $170 \mathrm{mg} / \mathrm{dl}$. The urinary sodium concentration was $12 \mathrm{mEq} / \mathrm{l}$. Further assessment of renal function showed a urine protein level of $8.2 \mathrm{~g} / \mathrm{l}$, a white cell number of $710,000 / \mathrm{ml}$ and a red cell number of $585,000 / \mathrm{ml}$; the presence of eosinophils, dysmorphic red cells and red cell casts was confirmed. The plasma albumin was determined to be $3.5 \mathrm{~g} / \mathrm{dl}(3.6-5.7 \mathrm{~g} / \mathrm{dl})$, and the globulin fraction was $1.5 \mathrm{~g} / \mathrm{dl}(0.5-1.28 \mathrm{~g} / \mathrm{dl})$. The renal ultrasound was normal.

All blood, tracheal secretions and urine cultures were sterile. Bacterial antigens and respiratory virus tests were also negative. Tests for signs of hemolysis and the presence of schizocytes were negative. The total complement and C3 fraction levels were low: 80\% (90-94\%) and 22.1 $\mathrm{mg} / \mathrm{dl}$ (90-180 mg/dl), respectively. The level of the complement component C4 was normal: $20.9 \mathrm{mg} / \mathrm{dl}(10-$ $40 \mathrm{mg} / \mathrm{dl}$ ). Assays for anti-nuclear antibody, anti-native DNA, cryoglobulins, ANCA (anti-neutrophil cytoplasmic antibodies) and anti-basement membrane antibody were negative. The level of anti-streptolysin O (ASO) was normal.

\section{Discussion}

In our clinical case, two important aspects are highlighted. The patient developed a case of respiratory insufficiency and renal dysfunction during treatment for an episode of community-acquired pneumonia (CAP). Acute respiratory insufficiency is one of the main reasons for admission in ICUs. Pneumonia is one of the most frequent causes of hypoxemic respiratory failure, leading to a requirement for mechanical ventilation. CAP is a serious condition that can have unfavorable outcomes including prolonged hospitalization, high costs and high mortality. It is believed that this disease is responsible for about 45,000 annual deaths in the USA. Refractory hypoxemia, persistent shock and the progression to multiple organ dysfunction (including AKI) are the main causes of death in individuals with CAP $[1,2]$.

The patient in question, despite initial improvement (the fever ceased and the expectoration decreased), developed a case of respiratory failure and needed mechanical ventilation. Despite an extensive microbiological investigation, the pathogen responsible for this event was not identified. In $40-70 \%$ of CAP cases, the etiology remains undetermined. Streptococcus pneumoniae is the most frequent cause of CAP, followed by other bacteria such as Haemophilus influenza, Klebsiella pneumoniae, Staphylococcus aureus, Legionella spp., Pseudomonas aeruginosa and viral infections.

In the evaluation of CAP, it is prudent to identify those individuals that will require hospitalization or intensive care. Some factors are indicators of the need for intensive care, such as the presence of comorbidities (diabetes, chronic kidney disease, chronic obstructive pulmonary disease), age ( $>60$ years old), clinical data (mental confusion, arterial hypotension, elevated respiratory rate) and laboratory data (acute renal dysfunction, bilateral pulmonary involvement in the diagnosis). The current guidelines recommend the combination of a $\beta$-lactam with coverage for pneumococci and a fluoroquinolone or a macrolide as initial treatment. In the presence of risk factors for infection by Pseudomonas (diabetes, bronchiectasis, neoplasia), S. aureus (diabetes, renal disease, infection with influenza) or resistant Pneumococcal (alcoholism, previous use of $\beta$-lactams, immunosuppression), directed antibiotic therapy must be instituted as soon as possible $[3,4]$.

Another important aspect in the clinical evolution of this patient was the development of acute renal dysfunction. In an interval of 1 week, the patient progressively became oliguric. The incidence of community-acquired AKI seems to be increasing in recent years. Pre-renal AKI and episodes of exacerbation of chronic kidney disease are the primary observed causes. In developed countries, pneumonia, urosepsis and the use of drugs (antibiotics, anti-retrovirals and non-hormonal anti-inflammatories) are the most common etiological factors leading to community-acquired AKI. AKI occurs in approximately $20 \%$ of CAP cases and also seems to be an independent factor for the risk of death. In developing countries, infectious causes (diarrhea, malaria, Dengue fever, leptospirosis), obstetric causes (septic abortion) and snake bites are still prevalent [5]. In the hospital environment, principally in the ICU, sepsis is the leading cause of AKI, and it fre- 
quently develops in the context of multiple organ dysfunction syndromes. These individuals are treated in ICUs and have a mortality of approximately $60 \%$ [6].

In the case presented here, the presence of AKI associated with signs of glomerular disease (proteinuria, hematuria and pyuria) suggests rapidly progressive glomerulonephritis. Glomerulonephritis is responsible for less than $10 \%$ of AKI cases. The association of rapidly progressive glomerulonephritis and pulmonary involvement (lung-kidney syndrome) suggests the diagnosis of vasculitis or anti-glomerular basement membrane antibodies disease (Goodpasture's disease). These individuals may develop anemia due to alveolar hemorrhage, which even when severe does not always manifest as hemoptysis. The radiological findings are not specific and show sparse or diffuse alveolar infiltrates. The presence of hemosiderinladen macrophages in bronchoalveolar lavage is suggestive of alveolar hemorrhage. A reduction of hemoglobin levels is common [7].

Small-vessel vasculitis, ANCA related mainly to Wegener's granulomatosis and microscopic polyangiitis are important causes of rapidly progressive glomerulonephritis. Both of these causes develop with segmental necrotizing glomerulonephritis, often with the formation of crescents and an absence of immune-deposits (pauciimmune). The blood levels of the complement system fractions are normal. Wegener's granulomatosis is most common in men, and in addition to rapidly progressive glomerulonephritis, it is characterized by pulmonary involvement (nodular and cavitary infiltrates, alveolar hemorrhage), superior respiratory tract involvement (sinusitis, nasopharyngeal ulcers), peripheral neuropathy, polyarthralgias and purpura. The main difference between these two diseases is the presence of granulomatous inflammation in Wegener's disease. While the majority of individuals with microscopic polyangiitis show anti-MPO (myeloperoxidase) ANCAs, $80-90 \%$ of cases of Wegener's granulomatosis show anti-PR3 (proteinase 3) ANCAs.

Patients with ANCA-associated vasculitis and renal involvement also produce ANCAs directed against human lysosomal membrane protein-2. The presence of eosinophilia and asthma distinguishes the Churg-Strauss syndrome from microscopic polyangiitis. In the case of Goodpasture's disease, the presence of anti-basal membrane antibodies (usually IgG) directed against the $\mathrm{NC1}$ domain of the $\alpha-3$ chain of type IV collagen (expressed in glomerular and alveolar basal membranes) is detected [8].

In this case, a negative ANCA and anti-basal membrane antibodies tests probably rule out the diagnosis of a small-vessel vasculitis ANCA-related and Goodpasture's disease, respectively. The low serum complement levels suggests the diagnosis of glomerulonephritis due to immune complex deposits. Negative antinuclear antibodies and circulating cryoglobulin tests exclude the diagnosis of lupus and cryoglobulinemia, respectively. Thus, the main hypothesis would be glomerulonephritis associated with pneumonia.

Provisional Diagnosis: Post-infectious glomerulonephritis (PIGN).

\section{Evaluation}

The patient became oliguric, and daily intermittent hemodialysis was initiated on the first day of admission to the ICU. On the second day of admission, an ultrasound-guided renal biopsy was performed.

\section{Histopathology Review}

Histological reports revealed a kidney with 16 glomeruli, of which three were globally sclerotic. The others were expanded in volume and were hypercellular due to infiltration of polymorphonuclear neutrophils with occlusion of the peripheral capillary loops. The podocytes exhibited moderate degenerative alterations. The presence of homogeneous scattered deposits in the subepithelial region ('humps') was noted. In two glomeruli, there were segmental cellular crescents. The tubules exhibited moderate degenerative epithelial alterations that were diffuse; some of the tubules had hyaline casts and cellular debris in the lumen, along with small foci of atrophy, edema, inflammatory infiltrates and interstitial fibrosis. Arterioles with sub-endothelial hyaline deposits and arterial vessels with moderate fibrous intimal hyperplasia were also present (fig. 1). Immunofluorescence showed granular deposits of IgG and C3 along the glomerular capillary walls and mesangium ('starry sky' pattern).

Methylprednisolone was administered $(1 \mathrm{mg} / \mathrm{kg})$ for 10 days. The patient remained dependent on mechanical ventilation for 8 days and on dialysis for 10 days. The patient was discharged from the ICU on the 11th day and discharged from the hospital on the 16th day with a creatinine concentration of $2.6 \mathrm{mg} / \mathrm{dl}$. After 6 years of evaluation, the patient's creatinine concentration was $1.7 \mathrm{mg} /$ $\mathrm{dl}$ (eGFR of $40 \mathrm{ml} / \mathrm{min}$ ), and the patient had a urine protein concentration of $0.3 \mathrm{~g} / \mathrm{l}$ in an isolated sample and normal urinary sediment. During this time, the patient became diabetic and had a coronary stent implanted. 


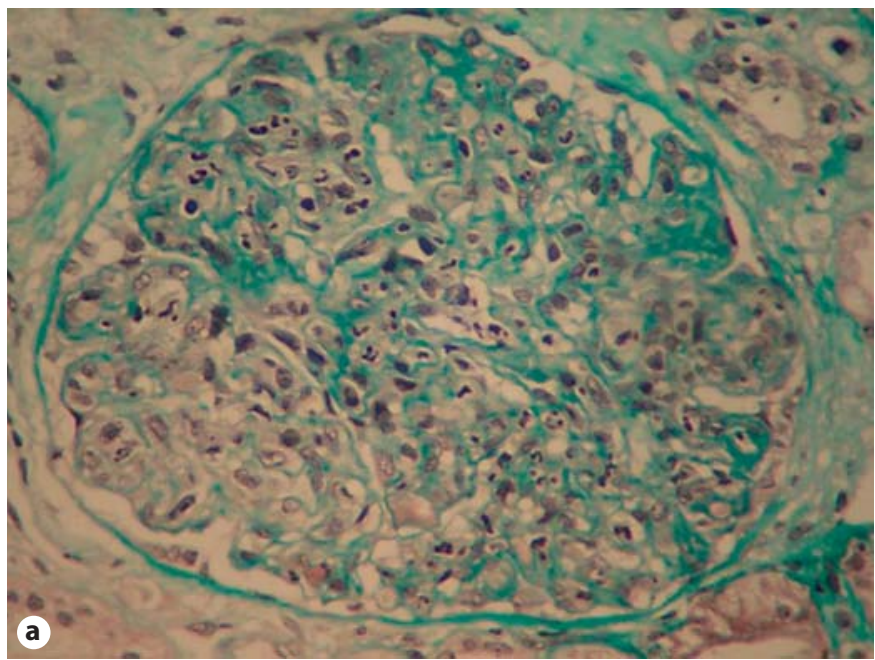

Fig. 1. Biopsy report: Masson's trichrome stain (glomerulus; a), hematoxilin and eosin stain (glomerulus; b), and Masson's trichrome stain (tubulus; c). Histological reports reveal a kidney with 16 glomeruli, of which 3 are globally sclerotic. The others are expanded in volume and hypercellular due to infiltration of polymorphonuclear neutrophils, with occlusion of the peripheral capillary loops. The podocytes exhibit moderate degenerative alterations. The presence of homogeneous scattered deposits in the subepithelial region ('humps') is noted. In two glomeruli, there are segmental cellular crescents. The tubules exhibit moderate degenerative epithelial alterations that are diffuse; some of the tubules have hyaline casts and cellular debris in the lumen, along with small foci of atrophy, edema, inflammatory infiltrates and interstitial fibrosis. Arterioles with sub-endothelial hyaline deposits and arterial vessels with moderate fibrous intimal hyperplasia are also present.

\section{Final Discussion}

The activation of the alternative pathway of the complement system associated with the histological picture of diffuse proliferation of endothelial and mesangial cells with intense neutrophilic exudate confirmed the diagnosis of PIGN. PIGN is an immunological response of the renal tissue against a distant infection usually caused by Lancefield group A $\beta$-hemolytic streptococcus (Streptococcus pyogenes). After 1-3 weeks of pharyngeal or skin infection, an acute nephritic case occurs. The renal tissue shows important endocapillary proliferation, neutrophilic exudate and varying degrees of immunoglobulin and complement deposits.

Difficulty in Breathing with Decreased Urine Output
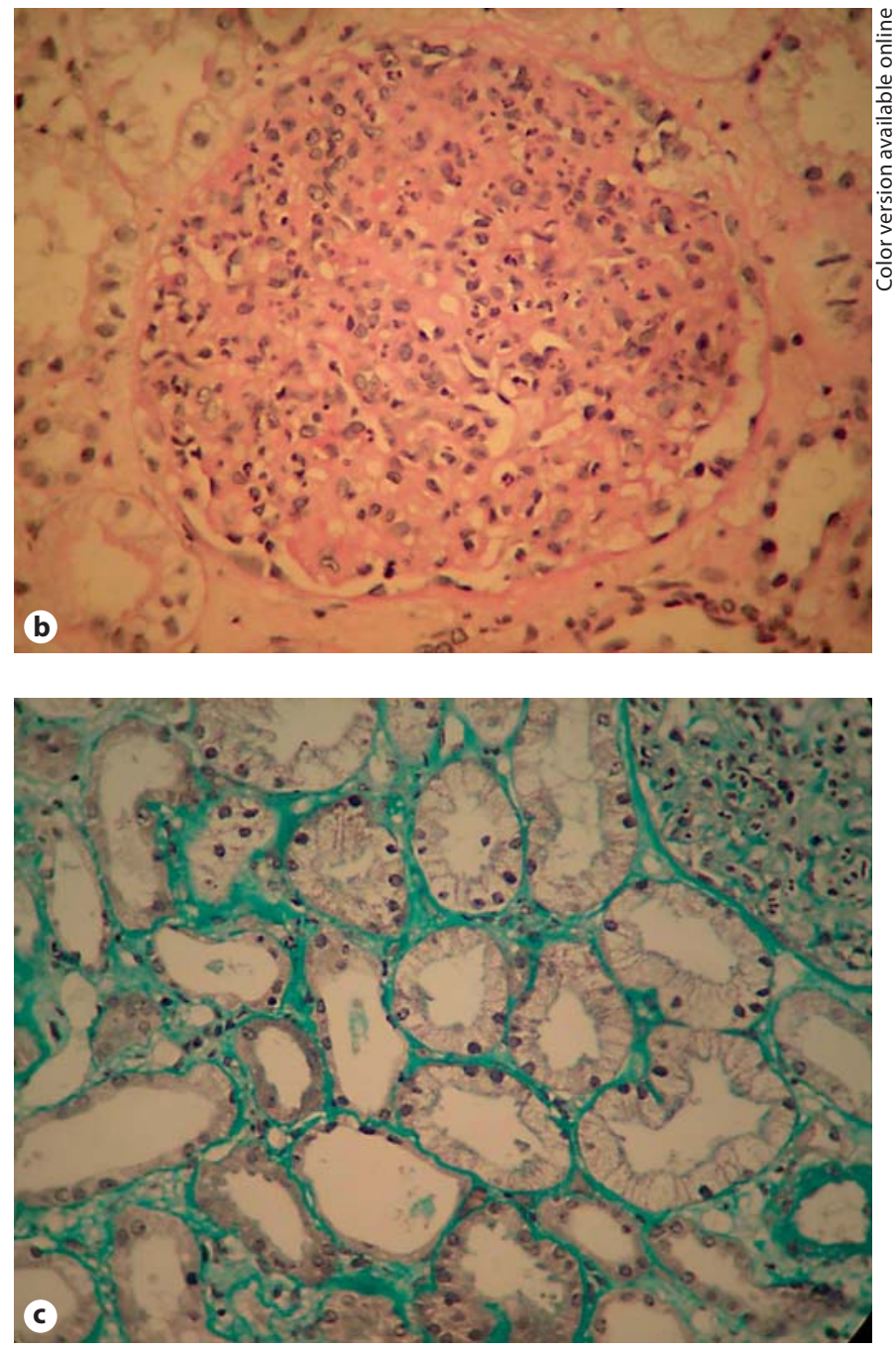

Clinically, the occurrence of hematuria, proteinuria and edema are often accompanied by arterial hypertension and mild renal insufficiency. In general, the disease has a benign character. Diuresis increases within 1 week and renal function normalizes in 2-4 weeks in parallel with histological improvements like reductions in the amount of inflammatory infiltrate and the number of immune-deposits. This disease principally affects children and young people from developing countries and is associated with a low socioeconomic level. Its overall incidence appears to be decreasing, and in some industrialized and developed regions, it has been practically eradicated. Despite the decrease in number of cases, epidemic outbreaks of PIGN still occur around the world. In Brazil for example, there was an outbreak in 1998 in the rural

Nephron Clin Pract 2010;116:c274-c282 
Table 1. Differential diagnosis of PIGN

IgA nephropathy
Lupus nephritis
Membranoproliferative glomerulonephritis
Bacterial endocarditis/visceral abscess/shunt nephritis
Anti-glomerular basement membrane disease
Amiloidosis due to chronic infection
Atheroembolic disease
Cryoglobulinemia
Small-vessel vasculitis ANCA-related
Acute interstitial nephritis
Hemolytic-uremic syndrome

region of Nova Serrana, state of Minas Gerais, caused by Streptococcus zooepidemicus originating from contaminated milk. One hundred thirty-five people developed glomerulonephritis, most of them adults. [9] Of the 56 individuals followed for 5 years, $57 \%$ showed some renal dysfunction [10].

In Latin America, infectious diseases like malaria, dengue, leptospirosis and diarrhea are an important cause of AKI in poor areas, while in urban areas, mainly in private and university hospitals, AKI profiles are similar to that observed in developed countries [11]. In Brazil, the largest Latin America country, most people live in urban areas and São Paulo is the largest South American city and many of the financial centers and industries are located there. In a Brazilian study almost two decades ago, chronic glomerulonephritis was the most common cause of end-stage renal disease [12]. Nowadays, the type 2 diabetes prevalence has been growing in most countries of Latin America [13]. In the Brazilian Peritoneal Dialysis Multicenter Study, diabetic nephropathy and renal vascular disease associated with hypertension were the more frequent causes of renal failure [14]. In 11 nephrology centers in the state of São Paulo, the most frequent secondary glomerular disease was lupus (66\% of cases), followed by PIGN (13\%) [15]. The differential diagnoses of PIGN are shown in table 1.

The main pathogenetic mechanism of PIGN seems to be the deposition of immune-complexes within the glomerular tuft. Currently, two antigens are being investigated as causes (nephritogenic) of PIGN: SpeB (streptococcal cationic proteinase exotoxin B) and GAPDH (streptococcal glyceraldehyde phosphate dehydrogenase). These antigens can interact with plasmin or plasminogen, activate collagenases and metalloproteinases and degrade the glomerular basal membrane, facili- tating the passage and the hump-like deposits of immune-complexes on the podocytary processes, which are characteristic of PIGN [16].

A wide range of microorganisms in addition to $S$. pyogenes are associated with the development of non-epidemic PIGN. The possible etiologies include bacteria, viruses, fungi, mycobacteria and parasitic infestations. In addition to skin and pharynx infections, endocarditis, pneumonias and visceral abscesses can also present with the occurrence of PIGN. Currently in developed countries, the cases of PIGN tend to occur in adults, especially in individuals with comorbidities such as diabetes and alcoholism. In a recent study with 86 patients (30\% diabetic) that had non-epidemic PIGN, mortality was 5\% and the evolution to end-stage renal disease was 17\% [17]. In another contemporary study, the majority (65\%) of 20 adult PIGN cases (average age of 61 years) needed dialysis support. Hypocomplementemia was present in $60 \%$ of the cases, and the most prevalent causative agent was $S$. aureus. During the follow-up period, six (30\%) individuals died and four (20\%) developed end-stage renal disease [18]. Therefore, PIGN in adults, and especially in the elderly, may have an unfavorable outcome, especially when it is associated with the formation of crescents and coexisting conditions such as diabetes and liver and cardiovascular diseases.

The treatment of PIGN is merely supportive. In streptococcal disease, the rapid eradication of bacteria through the use of antibiotics seems to prevent the transmission of nephritogenic strains during epidemic outbreaks, as well as reduce the incidence of glomerulonephritis.

When rapidly progressive glomerulonephritis is suspected, it is of paramount importance to initiate therapy with pulse corticosteroids and cy totoxic agents before the histopathologic investigation. In this patient specifically, it was not clear at the beginning if the respiratory failure was due to a pulmonary sepsis, so we decided to wait for the results of the biopsy, which was available in the following days. Treatment with corticosteroids and cytotoxic agents is questionable in individuals with PIGN, but might have a role in some circumstances [19], including a case with alveolar hemorrhage [20]. In our patient, we decided to try a short course of steroids $(1 \mathrm{mg} / \mathrm{kg}$ of methylprednisolone) because an adult with severe renal failure needing dialysis could be associated with the worst prognosis [18]. However, randomized clinical trials have not been performed to test steroids or other agents in this scenario. 


\section{Case Discussion}

Luis Y $u^{a}$, Miguel Cendoroglo Neto ${ }^{b}$, Bertrand L. Jaber', Eduardo Rocha ${ }^{d}$, Ronaldo Bergamo ${ }^{e}$, Renato Eick ${ }^{f}$, Ronaldo D'Avilag , José Tiburcio do Monte Neto ${ }^{h}$, Edilio Mattei', Waldemar Silva Almeida'

aFaculdade de Medicina Universidade de São Paulo, and ${ }^{b}$ Hospital Israelita Albert Einstein and Universidade Federal de São Paulo, São Paulo, Brazil; 'C Caritas St. Elizabeth's Medical Center and Tufts University School of Medicine, Boston, Mass., USA; 'Universidade Federal do Rio de Janeiro, Rio de Janeiro, eFaculdade de Medicina do ABC, Santo André, fHospital Moinho dos Ventos, Porto Alegre, 9Faculdade de Medicina da Pontifícia Universidade Católica de Sorocaba, Sorocaba, hUniversidade Federal de Piauí, Teresina, 'Hospital Israelita Albert Einstein, and jUniversidade Federal de São Paulo, São Paulo, Brazil

Luis Yu: What Is the Origin of Tubular Injury in This Situation?

Dr. de Souza Durão: In this case specifically, the patient did not use any potentially nephrotoxic drugs. At the time of hospitalization, the patient did not have arterial hypotension or any signs of dehydration. Although at the beginning it appeared to be an evolving pneumonic process, it subsequently was not a clear sepsis case. Sepsis, ischemia and nephrotoxicity are currently the primary causes of acute tubular necrosis. On the other hand, the patient was almost 60 -years-old, had hypertension and had an estimated basal glomerular filtration rate of $60 \mathrm{ml} / \mathrm{min}$. The biopsy showed arterial vessels with moderate fibrous intimal hyperplasia. These findings (advanced age, chronic kidney disease, arterial hypertension and vascular disease) are known predisposing factors for the development of acute tubular necrosis [21]. Furthermore, endocapillary proliferation determines glomerular ischemia. Leukocyte infiltration with the release of inflammatory mediators also reduces glomerular perfusion. Together, these factors can cause hypoperfusion of the peritubular capillaries and result in tubular ischemic injury accompanying glomerulonephritis. While not as abundant, the interstitial inflammatory infiltrate associated with edema is another potential factor contributing to tubular injury. The presence of red cell casts in the tubular lumen can result in direct cellular injury due to the toxicity of hemoglobin. Together, all these elements determine tubule-interstitial damage (including areas of tubular necrosis) associated with severe glomerulonephritis [22].

Difficulty in Breathing with Decreased Urine Output
Miguel Cendoroglo Neto: Specifically in This Case, Was the Alveolar Hemorrhage Diagnosis Clear?

Dr. de Souza Durão: The diagnosis of alveolar hemorrhage is performed in the clinical setting and is characterized by the triad of generally diffuse alveolar infiltrate, hemoptysis and anemia. It is frequently associated with vasculitis, collagen diseases, mitral stenosis, cocaine inhalation, use of some drugs (mitomycin C, propylthiouracil, etc.), bone marrow transplant and coagulopathies. These individuals present with increased diffusion of carbon monoxide due to the presence of hemoglobin in the alveoli [9]. Specifically in post-infectious acute glomerulonephritis, the case is complicated by pulmonary edema due to hypervolemia and the increased pulmonary capillary permeability induced by uremia and/or concomitant infectious respiratory processes. Our patient was anemic and showed hematic fluid by the orotracheal tube and bilateral alveolar infiltrate, which are suggestive of alveolar hemorrhage.

Bertrand L. Jaber: Is It Possible to Diagnose and Classify This Individual as Having AKI by the Acute Kidney Injury Network Criterion?

Dr. de Souza Durão: In the year 2003, the Acute Dialysis Quality Initiative (ADQI) group formulated the first consensus for the definition and classification of acute renal failure based on the RIFLE criterion. Later, in 2005, the Acute Kidney Injury Network (AKIN) proposed some modifications [23]. Now, AKI is considered to be an abrupt reduction (within $48 \mathrm{~h}$ ) of renal function, defined as an absolute increase of $0.3 \mathrm{mg} / \mathrm{dl}$ in serum creatinine or percentage increase of $\geq 50 \%$ of the basal value or a reduction in urine volume (documented oliguria $<0.5$ $\mathrm{ml} / \mathrm{kg}$ per hour for $>6 \mathrm{~h}$ ). This definition of AKI was conceived to be utilized in the hospital environment, but these temporal variations are difficult to document in individuals with community-acquired AKI. Therefore, the new definition of AKI has not been tested in settings outside of the hospital. In this case specifically, as we have the temporal evolution of the creatinine measurement (from 1.3 to $5.8 \mathrm{mg} / \mathrm{dl}$ in 8 days), we can extrapolate the concept and diagnose AKI according to the AKIN system.

Data validating the RIFLE and AKIN criteria in the evolution of glomerulonephritis are scarce in the literature. Recently, the RIFLE classification was predictive of progression and short-term prognosis of AKI in diffuse proliferative lupus nephritis [24]. 
Eduardo Rocha: What Should Be the Behavior of

Renal Injury Biomarkers in This Instance?

Dr. de Souza Durão: Recently, several biomarkers have been evaluated with the intent of early detection of AKI. Some of these substances are found in urine, usually in response to an ischemic and/or nephrotoxic injury. Examples of urinary biomarkers of AKI are: NGAL (neutrophil gelatinase-associated lipocalin), interleukin-18, KIM-1 (kidney injury molecule-1) and $\mathrm{NHE}_{3}$ (sodiumhydrogen exchanger isoform 3). Other substances are detected in the serum of patients early in the course of AKI, including cystatin C, NGAL, etc. $[25,26]$. The behavior of these biomarkers in glomerulonephritis is still unclear. It is known, for example, that the urinary levels of IL-18 are higher in patients with active nephritic syndrome [27]. The urinary levels of NGAL seem to correlate with the activity of lupus nephritis and in tubulointerstitial injury of glomerulopathy by $\operatorname{IgA}[28,29]$. The circulating levels of NGAL also correlated with flares of activity of ANCA-related vasculitis [30]. Therefore, some of these biomarkers may, in the future, become an additional tool for the diagnosis of activity and for the monitoring of treatments of various glomerulonephrities.

\section{Ronaldo Bergamo: Does the Absence of ASO Remove}

Streptococcal Infection as a Cause of the Process?

Dr. de Souza Durão: The identification of recent streptococcal infection can be accomplished by the detection of antibodies directed against antigens such as ASO, anti-streptokinase, anti-DNase and anti-hyaluronidase, among others. ASO titers are elevated in up to $80 \%$ of pharyngitis cases and in only $50 \%$ of impetigo cases. The early initiation of antibiotics can prevent the titers of these markers from increasing. Therefore, it is possible to have streptococcal infection without the elevation of ASO titers. Overall, the titers are elevated 1 week after acute infection, reach their peak around 2-4 weeks and normalize after 6-12 months. Pneumonia caused by group A hemolytic streptococcus is not common; historically, it occurs in women in their twenties and thirties, has a necrotizing character and is associated with large pleural effusions that progress to empyema, requiring thoracic drainage and often decortications of pleura [31].

\section{Renato Eick: Would the Presence of Eosinophiluria Be}

Suggestive of Acute Tubulointerstitial Nephritis?

Dr. de Souza Durão: Eosinophiluria is defined by the presence of more than $1 \%$ eosinophils of the total white cells in the urine. Detection of eosinophils might be done by different methods, but Hansel's stain is believed to be the best. Eosinophiluria is more frequently found in acute interstitial nephritis, but can also be present in other conditions like rapidly progressive glomerulonephritis, atheroembolic disease and in the infectious/inflammatory process like cystitis, prostatitis and pyelonephritis. However, failure to observe it does not discard the AIN diagnosis [32].

Ronaldo D'Avila: Could Chronic Glomerulonephritis

That Was Exacerbated in the Presence of the

Infectious Process Be the Cause?

Dr. de Souza Durão: The prognosis of PIGN is not necessarily benign. Some patients present with arterial hypertension, proteinuria, altered urinary sediment (hematuria) and sometimes evolve to end-stage renal disease. Perhaps some of these individuals may actually have membrane-proliferative glomerulonephritis. This disease can manifest through acute nephritic syndrome (similar to post-streptococcal glomerulonephritis), accidental discovery of hematuria and proteinuria in a routine laboratory exam, nephritic syndrome, or by recurrent episodes of hematuria during an episode of infection of the airways. Its idiopathic form occurs more commonly between the first and third decades of life. In these cases, histology shows a thickening of the glomerular basal membrane and prominent lobulation of glomeruli with deposition of eosinophilic material along the capillary wall [33]. The normalization of the complement level and of the urinary sediment excludes this possibility in our patient.

\section{José Tiburcio do Monte Neto: How Do the Levels of}

Complement Behave during the Course of the Disease?

Dr. de Souza Durão: Hypocomplementemia, especially low levels of $\mathrm{C} 3$, occurs during the first week of the disease in approximately $75 \%$ of patients. Generally, the levels of complement return to normal within 6 weeks. The hematuria is typically resolved between 3-6 months of evolution. The proteinuria has slower resolution during the recovery phase and may persist for years after the initial event [33].

\section{Edilio Mattei: How Is the Significant Proteinuria}

Justified in These Cases?

Dr. de Souza Durão: Proteinuria at nephrotic levels occurs in approximately $10 \%$ of individuals with post-streptococcal glomerulonephritis. The sub-epithelial deposits typical of PIGN (humps) activate the local complement system and lead to epithelial cellular injury, causing an increase in glomerular capillary permeability and subsequent proteinuria. The degree of proteinuria seems to be 
related to the number of these deposits, just as its clearance correlates with the resolution of protein extravasation. Humps are prominent during the first weeks of the disease and generally tend to disappear after the 6th week. Usually, the glomerular basal membrane is intact, although focal obliteration of the epithelial foot process may occur [34].

\section{Waldemar Silva Almeida: What Are the Mechanisms}

Involved in the Loss of Renal Function in PIGN?

Dr. de Souza Durão: In classic cases of post-streptococcal glomerulonephritis, the time between skin or pharynx infection and the development of nephritic syndrome is between 1 and 3 weeks. Approximately $25 \%$ of individuals present a significant reduction of glomerular filtration, and in some cases, the evolution will be rapidly progressive. Anuria is relatively uncommon, but some patients, especially adults, will require dialysis treatment. Severe acute renal failure is commonly associated with the formation of crescents. The oliguria generally resolves in 1 or 2 weeks $[35,36]$.

The endocapillary proliferation, the increased number of mesangial cells, the expansion of the mesangial matrix and the exogenous cellular infiltrate decrease the crosssectional area of the capillaries and compromise the glomerular blood flow. In experimental models of nephritis, there is a reduction of the glomerular ultrafiltration coefficient. The presence of crescents may obstruct the proximal tubules and stop glomerular ultrafiltration. Infiltration by inflammatory cells, especially neutrophils, leads to the production and release of substances such as proteases (elastase, cathepsin $\mathrm{G}$ ) and oxygen free radicals (hydrogen peroxide) that cause the degradation of the glomerular capillary wall. Local imbalances between the production of vasodilator and vasoconstrictor substances also compromise the glomerular plasma flow. Intra-glomerular thrombosis can also occur. All of these factors together are responsible for the reduced glomerular filtration [22].

\section{References}

1 Grossman RF, Rotschafer JC, Tan JS: Antimicrobial treatment of lower respiratory tract infections in the hospital setting. Am J Med 2005;118(Suppl 7A):29S-38S

$\checkmark 2$ Mortensen EM, Coley CM, Singer DE, Marrie TJ, Obrosky DS, Kapoor WN, Fine MJ: Causes of death for patients with community-acquired pneumonia: results from the Pneumonia Patient Outcomes Research Team cohort study. Arch Intern Med 2002; 162:1059-1064

3 File TM Jr, Tan JS, Plouffe JF: The role of atypical pathogens: Mycoplasma pneumoniae, Chlamydia pneumoniae, and Legionella pneumophila in respiratory infection. Infect Dis Clin North Am 1998;12:569-592.

4 Baddour LM, Yu VL, Klugman KP, Feldman C, Ortqvist A, Rello J, Morris AJ, Luna CM, Snydman DR, Ko WC, Chedid MB, Hui DS, Andremont A, Chiou CC, International Pneumococcal Study Group: Combination antibiotic therapy lowers mortality among severely ill patients with pneumococcal bacteremia. Am J Respir Crit Care Med 2004; 170:440-444

5 Lombardi R, Yu L, Younes-Ibrahim M, Schor N, Burdmann EA: Epidemiology of acute kidney injury in Latin America. Semin Nephrol 2008;28:320-329.

6 Durão MS, Monte JC, Batista MC, Oliveira M, Iizuka IJ, Santos BF, Pereira VG, Cendoroglo M, Santos OF: The use of regional citrate anticoagulation for continuous venovenous hemodiafiltration in acute kidney injury. Crit Care Med 2008;36:3024-3029.
7 Collard HR, Schwarz MI: Diffuse alveolar hemorrhage. Clin Chest Med 2004;25:583592.

8 Jennette JC: Rapidly progressive crescentic glomerulonephritis. Kidney Int 2003;63: 1164-1177.

-9 Balter S, Benin A, Pinto SW, Teixeira LM, Alvim GG, Luna E, Jackson D, LaClaire L, Elliott J, Facklam R, Schuchat A: Epidemic nephritis in Nova Serrana, Brazil. Lancet 2000; 355:1776-1780.

10 Sesso R, Pinto SW: Five-year follow-up of patients with epidemic glomerulonephritis due to Streptococcus zooepidemicus. Nephrol Dial Transplant 2005;20:1808-1812.

-11 Lombardi R, Yu L, Younes-Ibrahim M, Schor N, Burdmann EA: Epidemiology of acute kidney injury in Latin America. Semin Nephrol 2008;28:320-329.

12 Sesso R, Anção MS, Madeira SA: Epidemiologic aspects of the dialysis treatment in Grande São Paulo. Rev Assoc Med Bras 1994;40:10-14.

13 Cusumano AM, González Bedat MC: Chronic kidney disease in Latin America: time to improve screening and detection. Clin J Am Soc Nephrol 2008;3:594-600.

14 Fernandes N, Bastos MG, Cassi HV, Machado NL, Ribeiro JA, Martins G, Mourão O, Bastos K, Ferreira Filho SR, Lemos VM, Abdo M, Vannuchi MT, Mocelin A, Bettoni SL, Valenzuela RV, Lima MM, Pinto SW, Riella MC, Qureshi AR, Divino Filho JC, Pecoits-Filho R, Brazilian Peritoneal Dialysis Multicenter Study: The Brazilian Peritoneal
Dialysis Multicenter Study (BRAZPD): characterization of the cohort. Kidney Int Suppl 2008;108:S145-S151.

15 Malafronte P, Mastroianni-Kirsztajn GM, Betônico GN, Romão JE Jr, Alves MAR, Carvalho MF, Vieira Neto OM, Cadaval RAM, Bergamo RR, Woronik V, Sens YAS, Marrocos MSM, Barros RT: Paulista registry of glomerulonephritis: 5-year data report. Nephrol Dial Transplant 2006;21:3098-3105.

$>16$ Yoshizawa N: Acute glomerulonephritis. Intern Med 2000;39:687-694.

-17 Nasr SH, Markowitz GS, Stokes MB, Said SM, Valeri AM, D’Agati VD: Acute postinfectious glomerulonephritis in the modern era: experience with 86 adults and review of the literature. Medicine (Baltimore) 2008;87: 21-32.

$>18$ Wen YK: The spectrum of adult postinfectious glomerulonephritis in the new millennium. Ren Fail 2009;31:676-682.

19 Raff A, Hebert T, Pullman J, Coco M: Crescentic post-streptococcal glomerulonephritis with nephritic syndrome in the adult: is aggressive therapy warranted? Clin Nephrol 2005;63:375-380.

$>20$ Sung HY, Lim CH, Shin MJ, Kim BS, Song HC, Kim SY, Choi EJ, Chang YS, Bang BK: A case of post-streptococcal glomerulonephritis with diffuse alveolar hemorrhage. J Korean Med Sci 2007;22:1074-1078.

21 Gill N, Nally JV Jr, Fatica RA: Renal failure secondary to acute tubular necrosis: epidemiology, diagnosis, and management. Chest 2005;128:2847-2863. 
22 Pankewycz OG, Sturgill BC, Bolton WK: Proliferative glomerulonephritis: postinfectious, noninfectious and crescentic forms; in Tisher CC, Brenner BM (eds): Renal Pathology. Philadelphia, J B Lippincott Company, 1994, pp 2221-2257.

23 Mehta RL, Kellum JA, Shah SV, Molitoris BA, Ronco C, Warnock DG, Levin A, Acute Kidney Injury Network: Acute Kidney Injury Network: report of an initiative to improve outcomes in acute kidney injury. Crit Care 2007;11:R31.

24 Chen T, Ding X, Chen B: Value of the RIFLE classification for acute kidney injury in diffuse proliferative lupus nephritis. Nephrol Dial Transplant 2009;24:3115-3120.

-25 Parikh CR, Devarajan P: New biomarkers of acute kidney injury. Crit Care Med 2008; 36(4 Suppl):S159-S165.
26 Liangos $\mathrm{O}$, Tighiouart $\mathrm{H}$, Perianayagam MC, Kolyada A, Han WK, Wald R, Bonventre JV, Jaber BL: Comparative analysis of urinary biomarkers for early detection of acute kidney injury following cardiopulmonary bypass. Biomarkers 2009;14:423-431.

27 Matsumoto K, Kanmatsuse K: Elevated interleukin-18 levels in the urine of nephrotic patients. Nephron 2001;88:334-339.

28 Suzuki M, Wiers KM, Klein-Gitelman MS, Haines KA, Olson J, Onel KB, O'Neil K, Passo $\mathrm{MH}$, Singer NG, Tucker L, Ying J, Devarajan P, Brunner HI: Neutrophil gelatinase-associated lipocalin as a biomarker of disease activity in pediatric lupus nephritis. Pediatr Nephrol 2008;23:403-412.

29 Ding H, He Y, Li K, Yang J, Li X, Lu R, Gao W: Urinary neutrophil gelatinase-associated lipocalin (NGAL) is an early biomarker for renal tubulointerstitial injury in $\operatorname{IgA}$ nephropathy. Clin Immunol 2007; 123:227234.

30 Chen M, Wang F, Zhao MH: Circulating neutrophil gelatinase-associated lipocalin: a useful biomarker for assessing disease activity of ANCA-associated vasculitis. Rheumatology (Oxford) 2009;48:355-358.
31 Stevens DL: Streptococcal infections; in Goldman L, Ausiello D (eds): Cecil Medicine. Philadelphia, Saunders Elsevier, 2008, pp 2176-2182.

32 Michel DM, Kelly CJ: Acute interstitial nephritis. J Am Soc Nephrol 1998;9:506-515.

33 Tejani A, Ingulli E: Poststreptococcal glomerulonephritis. Current clinical and pathologic concepts. Nephron 1990;55:1-5.

34 Fries JW, Mendrick DL, Rennke HG: Determinants of immune complex-mediated glomerulonephritis. Kidney Int 1988;34:333345.

35 Rodriguez-Iturbe B, Musser JM: The current state of poststreptococcal glomerulonephritis. J Am Soc Nephrol 2008;19:1855-1864.

36 Kanjanabuch T, Kittikowit W, Eiam-Ong S: An update on acute postinfectious glomerulonephritis worldwide. Nat Rev Nephrol 2009;5:259-269. 